\title{
Genetic Polymorphisms and Cytokine Profile of Different Ethnicities in Septic Shock Patients, and their Association with Mortality
}

\author{
Shahla Siddiqui ${ }^{1}$, Resham Lal Gurung ${ }^{2}$, Sylvia Liu ${ }^{3}$, Edwin Chuen Ping Seet ${ }^{4}$, Su Chi Lim ${ }^{5}$
}

\begin{abstract}
Objective: The outcomes of sepsis and septic shock patients are heterogonous, with a variable response despite standardized care. The aim of this study was to explore the racial differences in septic shock outcomes, and their association with genetic polymorphisms and cytokine levels in an Asian population.

Design: This was an observational cohort study,

Setting: Intensive Care units of a 500 bedded tertiary care hospital in Singapore.

Patients: 198 patients (73 Chinese, 73 Malay and 52 Indian and others) admitted to the Khoo Teck Puat Hospital Intensive Care Unit between August 2016 and June 2017, with a diagnosis of severe sepsis (according to) were enrolled.

Interventions: Plasma interlukin-6 (IL-6), interlukin-10 (IL-10) and Tumour necrosis factor-a (TNFa) were measured using a highly sensitive quantitative sandwich enzyme-linked immunosorbent assay (ELISA) (BioVendor, Modrice, Czech Republic). The gene panel studied included 16 genes.

Measurements and Main Results: The rs7038903 common variant in SVEP1 gene showed significant association with sepsis severity independent of other variants in ordinal logistic and linear regression model ( $p=0.001$ and $p=0.002$ respectively). Moreover, the association between rs 7038903 and increased hazard for death remained significant after further adjusting for cytokines level. Interestingly, significant differences were seen in plasma IL6 in individuals with or without rs7038903 C allele (28pg/ml (IQR 12-86) vs 90pg/ml (IQR 49-155); P=0.022) in patients with severe sepsis in the Malay ethnic group.

Conclusions: Our study shows a promising polymorphism in SVEP1 gene (rs7038903) which is associated with sepsis shock and 28 days mortality, independent of age, gender, and method of diagnosis and SOFA score. Collectively, while our findings so far have shown the additional value or measuring cytokines and genetic markers in sepsis outcomes in the local population, further large scare studies are needed in a heterogeneous septic population with a rigorous analysis to know the significance of our findings.
\end{abstract}

Indian Journal of Critical Care Medicine (2019): 10.5005/jp-journals-10071-23136

\section{INTRODUCTION}

Sepsis and septic shock remain leading causes of death globally, with a mortality rate close to $50 \%{ }^{1}$. Sepsis is a multifactorial syndrome, with a complex pro-inflammatory response leading to high morbidity and mortality ${ }^{2}$. The outcomes of sepsis and septic shock patients are heterogonous, with a variable response despite standardized care ${ }^{3}$. Given the same treatment, some patients will recover and do well, whilst others will have poor outcomes. Several genes have been implicated after researchers turned their attention towards a more precise involvement of genetic variation ${ }^{4}$. Age, gender and ethnicity have been a focus of many studies in sepsis, but there is a large discrepancy in the results ${ }^{5}$. Racial variation in septic shock and mortality has been well documented. However, less is known about racial disparities among patients with sepsis and septic shock in a multi-ethnic Asian population. Singapore is composed of a predominantly Chinese population with a mix of Malay and Indians as well as foreigners. This ethnic mix produces an interesting cohort for such a study.

Cytokine and genomic studies can lead to early recognition and treatment of sepsis, as well as risk stratification ${ }^{6}$. Previous studies have inconsistently shown associations between genes and outcomes of sepsis ${ }^{7}$. Precision medicine studies have been changing practice for many diseases, and for an unpredictable and varied entity such as sepsis, it promises to be useful in the future ${ }^{8}$.

\footnotetext{
'Department of Anaesthesia, Khoo Teck Puat Hospital, Singapore

${ }^{2,5}$ Clinical Research Unit, Khoo Teck Puat Hospital, Singapore

3,4 Diabetes Centre, Khoo Teck Puat Hospital, Singapore.
}

Corresponding Author: Shahla Siddiqui, Anaesthesia and Intensive Care, Khoo Teck Puat Hospital, Singapore. Phone: 0065-6602 2317, Hp: 0065-97966257, -mail: shahlasi@yahoo.com

How to cite this article: Siddiqui S, Gurung RL et al. Genetic Polymorphisms and Cytokine Profile of Different Ethnicities in Septic Shock Patients, and their Association with Mortality. Indian J of Crit Care Med 2019;29(3):135-138.

Source of support: Nil

Conflict of interest: None

The aim of this study was to explore the racial differences in septic shock outcomes, and their association with genetic polymorphisms and cytokine levels in an Asian population.

\section{Materials and Methods}

\section{Study Population}

In this observational cohort study, 198 patients (73 Chinese, 73 Malay and 52 Indian and others) admitted to the Khoo Teck Puat Hospital Intensive Care Unit between August 2016 and June 2017, with a diagnosis of severe sepsis (according to) were enrolled.

(- The Author(s). 2019 Open Access This article is distributed under the terms of the Creative Commons Attribution 4.0 International License (http://creativecommons. org/licenses/by/4.0/), which permits unrestricted use, distribution, and non-commercial reproduction in any medium, provided you give appropriate credit to the original author(s) and the source, provide a link to the Creative Commons license, and indicate if changes were made. The Creative Commons Public Domain Dedication waiver (http://creativecommons.org/publicdomain/zero/1.0/) applies to the data made available in this article, unless otherwise stated. 
The subjects belonged primarily to the three major Asian ethnic groups of Singapore. Q Sofa score and SIRS score was used for inclusion criteria. Absence of bacteremia was an exclusion criterion. The primary outcome was 'septic shock', defined as "..profound circulatory, cellular, and metabolic abnormalities, associated with vasopressor requirement to maintain a mean arterial pressure of 65 $\mathrm{mm} \mathrm{Hg}$ or greater and serum lactate level greater than $2 \mathrm{mmol} / \mathrm{L}$ $\left(>18 \mathrm{mg} / \mathrm{dL}\right.$ ) in the absence of hypovolemia ${ }^{9}$." The secondary outcome was all-cause 28-day mortality.

The study was approved by the Singapore National Health Group Domain Specific Review Board and written consent was obtained from each participant or their legally acceptable representative.

\section{Cytokine Measurement}

Plasma interlukin-6 (IL-6), interlukin-10 (IL-10) and Tumour necrosis factor-a (TNFa) were measured using a highly sensitive quantitative sandwich enzyme-linked immunosorbent assay (ELISA) (BioVendor, Modrice, Czech Republic).

\section{Next-generation (Candidate Gene) Sequencing}

DNA was extracted from the buffy coat of blood samples using a QIAamp DNA maxi kit (Qiagen, Mississauga, ON, Canada). The gene panel studied included 16 genes; IL10, PCSK9, AMPD1, FCGR2A, HSP, MBP, LDLR, IL1, IL1R, IL1RN, HIF-1, CD14, TNF-alpha, IL6, TLR4, and SVEP1 (Table 1). Specific primers were designed using lon AmpliSeq Designer (Life Technologies, USA), covering all exons and 100 base pair of exon intron junction. The exome library was prepared using the Ion AmpliSeq Library Kit (Thermo Fisher Scientific, USA) and sequenced using lon PGM Hi-Q Sequencing Kit and ' 318 ' sequencing chips on lon Torrent PGM System (Thermo Fisher Scientific, USA). Analyses were performed using Torrent Suite and lon Reporter (Thermo Fisher Scientific, USA). Sequence reads were aligned to the human GRC37/hg19.

\section{Variant Calling}

Variants were filtered to exclude coverage depth of $<100$ reads, synonymous variants. Only non-synonymous variant were selected for subsequent analysis. Functional prediction of variants were assessed with mutation prediction tools, including PolyPhen2 [17], SIFT [18], and MutationTaster2 [19], which are within the Alamut software (REF). Only non-synonymous variant predicted to be functionally deleterious by at least two bioinformatics tools were selected for subsequent analysis.

\section{Statistical Analysis}

Continuous variables were expressed as median and interquartile ranges (IQR). Categorical data were expressed as proportions. Participants were stratified by ethnicity and outcomes (septic shock and 28 days mortality status) to visualize clinical and biochemical characteristics. We used the Kruskal-Wallis test to test for differences in baseline characteristics for continuous data and $x 2$ test for categorical variables. The association between cytokine levels and sepsis shock were evaluated using logistic regression model in all samples and separately by ethnic group. Non-normally distributed data were natural log-transformed to obtain normal distributions. Model was adjusted for demographic variables age, gender, surgical method and Qsofa. The association between cytokine levels and 28-days mortality were evaluated using Cox regression analysis adjusting for baseline age, gender, surgical method and Qsofa.

For association of genotype with septic outcomes, we first performed regression analysis (additive model) for association of 53 SNPs identified with septic shock and 28-day mortality. Given our small sample size in the study, we also defined sepsis severity outcome as severe sepsis, septic shock and uses of vasopressin and mortality within 28 days and performed 1) ordinal logistic regression analysis and 2) linear regression analysis. SNP with suggestive evidence of association with either of three outcomes were selected for subsequent multivariate association analyses. The Cox regression was used to test for differences in the hazard of death over 28 days according to genotype, adjusting for age, gender, a surgical versus a medical primary diagnosis, ethnicity, and qSOFA score. P value less than 0.05 were considered statistically significant. All analyses were performed using SPSS version 22 (SPSS Inc, IBM Corporation, Chicago, IL) and STAT/ SE 14.0 (StataCorp, College Station, Texas).

Table 1: Baseline profile of subjects stratified by ethnicity

\begin{tabular}{lllll}
\hline Characteristics & $\begin{array}{l}\text { Chinese } \\
(n=73)\end{array}$ & $\begin{array}{l}\text { Malay } \\
(n=73)\end{array}$ & $\begin{array}{l}\text { Indian } \\
(n=52)\end{array}$ & P-value \\
\hline Age (years) & $63.0(49.5-73.5)$ & $54.0(45.0-64.0)$ & $61.0(40.0-68.8)$ & 0.044 \\
$\quad>65$ years $(\mathrm{n}, \%)$ & $31(42.5)$ & $14(19.2)$ & $17(27.9)$ & 0.010 \\
Female $(\mathrm{n}, \%)$ & $29(39.7)$ & $28(38.4)$ & $21(27.3)$ & 0.972 \\
BMI $\left(\mathrm{kg} / \mathrm{m}^{2}\right)$ & $22.1(19.4-22.3)$ & $27.1(23.3-32.0)$ & $22.6(20.5-27.5)$ & $<0.001$ \\
Obese $\left(\mathrm{BMl}>27.5 \mathrm{~kg} / \mathrm{m}^{2}\right)(\%)$ & 12.7 & 47.0 & 24.4 & $<0.0001$ \\
SBP $(\mathrm{mmHg})$ & $123(110-138)$ & $126(103-139)$ & $119(106-134)$ & 0.601 \\
DBP $(\mathrm{mmHg})$ & $69(61-78)$ & $70(59-82)$ & $67(57-79)$ & 0.594 \\
qSOFA & $2.0(2.0-2.5)$ & $2.0(2.0-3.0)$ & $2.0(2.0-2.0)$ & 0.296 \\
Cytokines & & & & \\
$\quad$ IL6 $(\mathrm{pg} / \mathrm{ml})$ & $51.8(13.1-198.7)$ & $43.2(13.9-98.5)$ & $29.4(13.9-59.9)$ & 0.261 \\
IL10 $(\mathrm{pg} / \mathrm{ml})$ & $13.3(7.0-28.8)$ & $12.8(7.7-25.7)$ & $12.0(6.5-23.6)$ & 0.744 \\
$\quad$ TNF-a $(\mathrm{pg} / \mathrm{ml})$ & $7.7(3.5-17.3)$ & $7.4(3.6-14.0)$ & $5.8(3.8-14.2)$ & 0.873 \\
Outcome $(n, \%)$ & & & & \\
$\quad$ Sepsis shock & $50(68.5)$ & $44(60.3)$ & $30(57.7)$ & 0.409 \\
$\quad 28$ days Mortality & $9(12.3)$ & $8(11.1)$ & $5(9.8)$ & 0.896 \\
Length of Stay (days) & $5.5(2.0-10.0)$ & $4.0(3.0-8.5)$ & $5.0(3.0-8.8)$ & 0.907 \\
\hline
\end{tabular}

Data are presented as frequency (\%) for categorical variables or median (25th-75th percentile).

Abbreviations: BMI, body mass index; SBP, systolic blood pressure; DBP, diastolic blood pressure; IL6, interlukin-6; IL10, interlukin-10; TNF-a, tumour necrosis factor alpha. 


\section{Results}

Table 1 displays the baseline profile of the participants by ethnicity. Malay patients (median age 54.0, IQR 45-63) admitted were on average 9 years and 7 years younger than Chinese and Indian patients respectively $(p=0.033)$ and the proportion of younger patients $(<65$ years) were significantly higher $(p=0.006)$ in Malay (18\%) as compared to Indian (28\%) and Chinese (43\%) (Table 1). $\mathrm{BMI}$ and proportion of obese patients were also higher in Malay compared to Chinese and Indian ( $p<0.001)$.The incidence of septic shock was comparable across ethnic groups (68.5\% in Chinese, $61.1 \%$ in Malay and $61.5 \%$ in Indian, $p=0.421$ ). However, relative to non-septic shock patients, significant increase in both proinflammatory cytokines IL- 6 and TNFa in septic shock patients was observed only in Malay ( $p=0.008, p=0.002$ ) (Table 2).

\section{Association of Cytokines and Septic Outcomes}

Binary logistic regression analysis stratified by ethnicity and adjusted for age and gender revealed greater risk of septic shock associated with increased cytokines levels in Malay compared to Chinese or Indian. The 28 days all-cause mortality rate was between 10 to $12 \%$ in three different ethnic group. Malay patients with increased IL- 6 and IL-10 levels had elevated risk for all-cause mortality in the cox regression analysis (hazard ratio $(\mathrm{HR})=2.45,95 \%$ $\mathrm{Cl} 0.95-6.36, \mathrm{p}=0.065$ for natural log transformed IL 6 and $\mathrm{HR}=3.26$, $95 \% \mathrm{Cl} 1.19-8.95, \mathrm{p}=0.022$ for natural log transformed IL10) (Table 3).

\section{Genetic Profiling}

In our study, we focused on non-synonymous single-nucleotide polymorphisms (SNPs) that disrupt conserved regions of the genome and are predicted to have significant effects on protein function based on in silico bioinformatics tools. Functional prediction was assessed by four different bioinformatics algorithm* (Mutation Taster, GVGD, SIFT and PolyPhen-2). Only variants predicted to have deleterious effect on the function of protein by 2 or more tools were selected. Accordingly, we had identified 53 variants, of which 4 were common variants (Minor allele frequency $(\mathrm{MAF})>5 \%), 2$ variants with $5 \%>M A F>1 \%$ and remaining extremely rare. To identify variants potentially associated with sepsis outcomes, we undertook several strategy given our modest sample size in this pilot study. We examined the association of variants with sepsis severity (severe sepsis, septic shock and mortality) in ordinal logistic and linear regression model and identify specific SNPs with suggestive association. The rs7038903 common variant in SVEP1 gene showed significant association with sepsis severity independent of other variants in ordinal logistic and linear regression model ( $p=0.001$ and $p=0.002$ respectively). According to Exome Aggregation Consortium (ExAC), minor allele frequency of rs7038903 is reported to be approximately $8 \%$ and $14 \%$ in East Asian and South Asian respectively. We observed similar MAF for rs7038903 (6-8\%) in our study population.

\section{Association of rs7038903 and Septic Outcomes}

We next tested for the association of rs7038903 with septic shock and 28 days mortality adjusting for clinical covariates. Patients with Callele of rs7038903 were at increased risk for development of septic shock $(\mathrm{OR}=2.4495 \% \mathrm{Cl} 0.96-6.21 ; \mathrm{P}=0.060)$ as compared to patients with T allele. This increased risk remained after adjustment for age, gender, ancestry, surgical methods and qSOFA scores (OR=2.58;

Table 2: Association of cytokine levels with development of Septic shock and 28 days mortality stratified by ethnicity

\begin{tabular}{|c|c|c|c|c|c|c|c|c|c|}
\hline & & Chinese & & Malay & & Indians & & All samples & \\
\hline Outcomes & Variable & OR $(95 \% C l)$ & Pvalue & OR $(95 \% C l)$ & Pvalue & OR $(95 \% C l)$ & Pvalue & OR $(95 \% \mathrm{Cl})$ & Pvalue \\
\hline \multirow[t]{3}{*}{ Septic shock } & $\mathrm{IL}-6^{\ddagger}$ & $1.65(1.12-2.42)$ & 0.011 & $1.82(1.20-2.76)$ & 0.005 & $1.67(0.94-2.97)$ & 0.083 & $1.60(1.27-2.02)$ & $<0.0001$ \\
\hline & $\mathrm{IL}-10^{\ddagger}$ & $2.59(1.40-4.81)$ & 0.003 & $2.33(1.16-4.69)$ & 0.018 & $1.97(0.94-4.11)$ & 0.072 & $2.18(1.50-3.17)$ & $<0.0001$ \\
\hline & $\mathrm{TNFa}^{\ddagger}$ & $2.01(1.23-3.28)$ & 0.005 & $2.45(1.36-4.42)$ & 0.003 & $1.47(0.98-3.19)$ & 0.327 & $1.96(1.43-2.68)$ & $<0.0001$ \\
\hline \multirow[t]{4}{*}{28 days mortality } & & $\mathrm{HR}(95 \% \mathrm{Cl})$ & Pvalue & $\mathrm{HR}(95 \% \mathrm{Cl})$ & Pvalue & $\mathrm{HR}(95 \% \mathrm{Cl})$ & Pvalue & $\mathrm{HR}(95 \% \mathrm{Cl})$ & Pvalue \\
\hline & $\mathrm{IL}-6^{\ddagger}$ & $1.12(0.67-1.87)$ & 0.659 & $2.13(1.16-9.93)$ & 0.015 & $3.12(0.78-12.5)$ & 0.107 & $1.46(1.11-1.92)$ & 0.008 \\
\hline & $\mathrm{IL}-10^{\ddagger}$ & $0.99(0.46-2.12)$ & 0.977 & $3.19(1.31-7.72)$ & 0.010 & $1.60(0.66-3.87)$ & 0.300 & $1.53(1.08-2.17)$ & 0.016 \\
\hline & $\mathrm{TNFa}^{\ddagger}$ & $0.681(0.34-1.37)$ & 0.283 & $3.22(1.33-7.78)$ & 0.009 & $2.56(0.44-15.06)$ & 0.299 & $1.62(1.01-2.60)$ & 0.046 \\
\hline
\end{tabular}

Septic shock and 28 days mortality was measured by logistic regression and cox regression respectively adjusted for age, gender, surgical method, Qsofa and ethnicity (in all samples). ${ }^{\ddagger}$ Natural log transformed. $\mathrm{P}<0.05$ was presented in bold.

Table 3: Association of SNP with development of Septic shock and 28 days mortality

\begin{tabular}{llllll}
\hline & \multicolumn{3}{l}{ Septic Shock } & \multicolumn{3}{l}{ Mortality } \\
\cline { 2 - 6 } & Variable & OR $(95 \% \mathrm{Cl})$ & Pvalue & $H R(95 \% \mathrm{Cl})$ & P value \\
\hline Unadjusted & rs7038903 & $2.44(0.96-6.21)$ & 0.060 & $2.14(0.91-5.02)$ & 0.080 \\
Adjusted & Age & $1.02(1.00-1.04)$ & 0.034 & $1.00(0.97-1.02)$ & 0.796 \\
& Female vs Male & $0.69(0.37-1.28)$ & 0.241 & $1.08(0.44-2.64)$ & 0.866 \\
& Indian vs Chinese & $0.66(0.30-1.43)$ & 0.289 & $0.75(0.25-2.27)$ & 0.604 \\
& Malay vs Chinese & $0.68(0.33-1.41)$ & 0.303 & $0.74(0.28-1.94)$ & 0.548 \\
& Medical vs surgical & $1.00(0.54-1.86)$ & 0.994 & $1.35(0.52-3.48)$ & 0.540 \\
& qSOFA & $\mathbf{1 . 6 3 ( 1 . 0 5 - 2 . 5 3 )}$ & $\mathbf{0 . 0 3 0}$ & $\mathbf{5 . 1 0 ( 2 . 2 3 - 1 1 . 6 8 )}$ & $<0.001$ \\
& rs7038903 & $\mathbf{2 . 5 8 ( 0 . 9 8 - 6 . 8 1 )}$ & $\mathbf{0 . 0 5 6}$ & $\mathbf{2 . 9 6 ( 1 . 1 0 - 7 . 9 4 )}$ & $\mathbf{0 . 0 3 1}$ \\
\hline
\end{tabular}

Septic shock and 28 days mortality was measured by logistic regression and cox regression respectively adjusted for age, gender, surgical method, Qsofa and ethnicity (in all samples). $\mathrm{P}<0.05$ was presented in bold. 
95\%Cl 0.98-6.81; $\mathrm{P}=0.056)$. We also observed that patients with rs7038903 had a significantly increased hazard of death over the 28 days observation independent of age, gender, ancestry, surgical method and qSOFA score in the Cox regression model (adjusted HR 2.96; $95 \% \mathrm{Cl} 1.10-7.94 ; \mathrm{P}=0.031)$. Moreover, the association between rs7038903 and increased hazard for death remained significant after further adjusting for cytokines level. Interestingly, significant differences were seen in plasma IL6 in individuals with or without rs7038903 C allele (28pg/ml (IQR 12-86) vs $90 \mathrm{pg} / \mathrm{ml}$ (IQR 49-155); $\mathrm{P}=0.022$ ) in patients with severe sepsis in the Malay ethnic group.

\section{Discussion}

The incidence of septic shock and mortality were similar regardless of race. However, there were notable racial disparities in association of cytokines level and septic shock and all-cause mortality in Asian population. Further studies in larger Asian populations are warranted to validate our findings. Although age in general is a risk factor for poor outcomes in sepsis, significant difference in age was observed among various ethnicities. The proportion of septic young patients with poor outcomes (less than 65 years old) was highest among Malays.

SVEP1 is similar to the selectin family of adhesion proteins and was previously proven to be a cell surface protein involved in cell adhesion processes. A recent study demonstrates that SVEP1 is a ligand for integrin $\alpha 9 \beta 1$ and mediates cell adhesion protein is composed of 3,571 amino acids having multiple structural domains including Sushi domain, von Willibrand factor type A domain, epidermal growth factor domain, hyaline repeat domain, and pentraxin domains SVEP1 expression detected in the tissues of heart, lung, skeletal tissue, placenta ,stomach, intestine, stromal osteogenic tissues (periosteum and bone), and bone marrow mesenchymal stromal cells, endothelial cells, and breast carcinoma cells $s^{10}$. Sushi domains are short consensus repeats belonging to the family of complement control proteins (CCPs). CCP protein modules are of approximately 60 amino acids and have protein-protein interaction domains with many other proteins that regulate blood coagulation and complement systems. Our study shows a promising polymorphism in SVEP1 gene (rs7038903) which is associated with sepsis shock and 28 days mortality, independent of age, gender, and method of diagnosis and SOFA score. Collectively, while our findings so far have shown the additional value or measuring cytokines and genetic markers in sepsis outcomes in the local population, further large scare studies are needed in a heterogeneous septic population with a rigorous analysis to know the significance of our findings.
To our best knowledge, our study is the first to perform genotype profiling in severe sepsis population in Singapore and prospectively determine the association with sepsis shock and mortality. Furthermore, we used multiple mutation prediction analysis tools used for function prediction to identify the variant with deleterious impact. Several limitations of our study should be acknowledged. Firstly, we did not correct for multiple genotyping comparisons given our modest sample size. Furthermore, a replication in other sepsis population would provide more confidence to our data. While we used multiple analysis tool to predict functional impact of rs7038903, further function studies using knock out models would verify the causality.

\section{References}

1. Singer M, Deutschman CS, Seymour CW, et al. The Third International Consensus Definitions for Sepsis and Septic Shock (Sepsis-3). JAMA. 2016;315(8):801-810. doi:10.1001/jama.2016.0287

2. Dellinger RP, Levy MM, Rhodes A, et al; Surviving Sepsis Campaign Guidelines Committee Including the Pediatric Subgroup. Surviving Sepsis Campaign: international guidelines for management of severe sepsis and septic shock: 2012. Crit Care Med. 2013;41(2):580-637

3. Iskander KN, Osuchowski MF, Stearns-Kurosawa DJ, et al. Sepsis: Multiple Abnormalities, Heterogeneous Responses, and Evolving Understanding. Physiological Reviews. 2013;93(3):1247-1288. doi:10.1152/physrev.00037.2012.

4. Sutherland AM, Walley KR. Bench-to-bedside review: Association of genetic variation with sepsis. Critical Care. 2009;13(2):210. doi:10.1186/ cc7702.

5. Barnato AE, Alexander SL, Linde-Zwirble WT, Angus DC. Racial Variation in the Incidence, Care, and Outcomes of Severe Sepsis: Analysis of Population, Patient, and Hospital Characteristics. American Journal of Respiratory and Critical Care Medicine. 2008;177(3):279-284. doi:10.1164/rccm.200703-4800C.

6. Frank Stüber, Sven Klaschik, Lutz Eric Lehmann, Jens-Christian Schewe, Stefan Weber, Malte Book; Cytokine Promoter Polymorphisms in Severe Sepsis, Clinical Infectious Diseases, Volume 41, Issue Supplement_7, 15 November 2005, Pages S416-S420

7. Stuber F, Petersen M, Bokelmann F, Schade U. A genomic polymorphism within the tumor necrosis factor locus influences plasma tumor necrosis factor-alpha concentrations and outcome of patients with severe sepsis, Crit Care

8. Wang Z-G, Zhang L, Zhao W-J. Definition and application of precision medicine. Chinese Journal of Traumatology. 2016;19(5):249-250. doi:10.1016/j.cjtee.2016.04.005.

9. Nakada TA1, Russell JA, Boyd JH, Thair SA, Walley KR. Identification of a nonsynonymous polymorphism in the SVEP1 gene associated with altered clinical outcomes in septic shock. Crit Care Med. 2015 Jan;43(1):101-8. 